\title{
Photovoltaic light valving induced in a vertically aligned nematic liquid crystal on a $x$-cut Fe:LiNbO ${ }_{3}$ substrate
}

https://doi.org/10.1515/odps-2018-0002

Received June 5, 2018; revised September 11, 2018; accepted September 22, 2018

\begin{abstract}
Photovoltaic fields induced in $x$-cut Fe-doped lithium niobate $\left(\mathrm{Fe}: \mathrm{LiNbO}_{3}\right.$ ) were used to achieve optically induced defect formation and light valving in a vertically aligned nematic liquid crystal. Initially, the optical axis of the LC was vertically aligned (along the surface-normal of the planar, photovoltaic substrates) throughout the whole sample. Samples were exposed with a focused continuous wave laser beam and investigated via microscopic imaging in-between crossed polarizers. The optical axis of the planar, $x$-cut $\mathrm{Fe}: \mathrm{LiNbO}_{3}$ substrates was in the substrate plane and oriented parallel to one of the polarizers, which resulted in an initially dark state. Optically induced surface fields (with high in-plane components) generated within the substrates led to director reorientations and defect formation. Accordingly, the samples were locally switched into a transmissive state. The area affected by exposure was larger $(300 \mu \mathrm{m})$ than the FWHM of the Gaussian exposure beam $(14 \mu \mathrm{m})$. Switching from dark to bright states (light valving) could be achieved in the investigated samples much more efficiently than in previously investigated samples with $z$-cut Fe: $\mathrm{LiNbO}_{3}$-substrates. Realignments of the LC director were induced at lower optical power density $\left(140 \mathrm{~mW} / \mathrm{cm}^{2}\right)$ than would be required to excite the intrinsically present nonlinear optical responses in a nematic LC such as the light induced Fredericks transition.
\end{abstract}

Keywords: opto-optic responses, nonlinear optical responses, soft matter, optical modulators

\section{Introduction}

Localized and patterned exposure of Fe: $\mathrm{LiNbO}_{3}$ substrates has been investigated, particularly, for optoelectronic tweezers for manipulation of dielectric particles (Arregui et al., 2015; Cándido Arregui et al., 2014; Carrascosa, García-Cabañes, Jubera, Ramiro, \& Agulló-López, 2015; Carrascosa et al., 2015; Esseling et al., 2013; GarcíaCabañes et al., 2018; Gazzetto et al., 2016; Villarroel et al., 2011) and fluid substances (Fan et al., 2017; Habibpourmoghadam, Jiao, Reshetnyak, Evans, \& Lorenz, 2017). Due to their high birefringence and large electrooptic responses, liquid crystals (LCs) are highly sensitive for surface fields induced in $\mathrm{Fe}: \mathrm{LiNbO}_{3}$. Localized defect creation (Habibpourmoghadam, Lucchetti, et al., 2017; Habibpourmoghadam, Jiao, et al., 2017), lensing (Habibpourmoghadam, Jiao, et al., 2017), defect-free director realignments ( Carns et al., 2006; Carns et al., 2006; Lucchetti et al., 2017; Lucchetti, Kushnir, Ciciulla, et al., 2016; Lucchetti, Kushnir, Zaltron, \& Simoni, 2016), and erasable pattern formation (Habibpourmoghadam, Lucchetti, et al., 2017) have been reported, recently. Field induced realignments of the local optical axis (director realignment) in LC samples is easily detected via imaging or via the modulation of probe beams. However, $\mathrm{LiNbO}_{3}$ also is a birefringent material and the combination of a crystalline and a liquid crystalline birefringent optical media in a hybridized sample can be challenging. In previous studies (Carns et al., 2006; Carns et al., 2006; Habibpour-

\footnotetext{
Lin Jiao: Department of Chemistry, Paderborn University, Warburger Str. 100, 33098 Paderborn, Germany Dean R. Evans: Air Force Research Laboratory, Materials and Manufacturing Directorate, Wright-Patterson Air Force Base, Ohio 45433, USA

*Corresponding Author: Alexander Lorenz: Department of Chemistry, Paderborn University, Warburger Str. 100, 33098 Paderborn, Germany and Institute of Chemistry, FB10, University of Kassel, HeinrichPlett-Str. 40, 34132 Kassel, Germany, E-mail: alexander.lorenz@online.ms
} 
moghadam, Lucchetti, et al., 2017; Habibpourmoghadam, Jiao, et al., 2017; Lucchetti et al., 2017; Lucchetti, Kushnir, Ciciulla, et al., 2016; Lucchetti, Kushnir, Zaltron, et al., 2016), $z$-cut Fe: $\mathrm{LiNbO}_{3}$ substrates were used very efficiently to achieve LC realignments, and the generated electric field distribution was investigated in detail. There, the substrate's optical axis was parallel to the substrate's layer normal, and the field distribution of photovoltaic fields (bipolar fields) had axial symmetry $\left(C_{\infty}\right)$. Such fields led to centro-symmetric LC reorientations $\left(C_{\infty}\right)$ in vertically aligned LCs and hyperbolic defects with reduced $\left(C_{4}\right)$ rotational symmetry in samples where the LC initially had uniform orientation, perpendicular to the layer normal of the substrates, which reduced the symmetry of the LC realignment, eventually causing formation of line defects.

In the current work, samples were prepared with $x$-cut $\mathrm{Fe}: \mathrm{LiNbO}_{3}$ substrates (polar axis in the substrate plane) and the LC had vertical alignment (the optical axis of the LC was aligned parallel to the layer normal of the substrates). Exposure with a focused continuous wave laser beam and imaging of the LC reorientation revealed formation of a defect pattern within the LC layer, where several line defects were induced. From the patterns, it could be deduced that the LC director locally was parallel to the electric fields. The LC reorientations expanded in an area much larger than the beam focus.

Photographs of $x$-cut Fe:LiNbO 3 substrates were recorded in-between crossed polarization filters (Fig. 1a). As seen in the figure, the substrate was dark, if the substrate's optical axis was parallel to one of these polarizers. As expected, the substrate appeared bright when rotated such as its optical axis was no more parallel to the polarization direction of the incident light, (Fig. 1b). In ferroelectrics with high anomalous photovoltaic response, like Fe: $\mathrm{LiNbO}_{3}$, exposure to light can generate high internal electric fields and also photovoltaic (near-surface) fields. These light induced fields are generated by a photoinduced charge carrier separation within the substrates, parallel to their polar, crystallographic $c$-axis. These fields are caused by optically induced drift of electrons (GarcíaCabañes et al., 2018; Lucchetti et al., 2017; Volk, 2010). The resulting photovoltaic fields were calculated in both $z$-cut and $x$-cut substrates (Arregui et al., 2014) for patterned illumination and for exposure to a Gaussian laser beam. As shown in [(Arregui et al., 2014)], generation of fringe in-plane fields can be expected in $x$-cut substrates. These fields expand beyond the beam waist of the Gaussian exposure beam. A schematic of the generated field distribution is shown in Fig. 2. Here, the charge carrier separation is induced parallel to the substrate's polar $c$ axis. The photovoltaic field (near surface field) can be de- scribed as dipolar field. The FWHM (full width at half maximum) of the Gaussian exposure beam is indicated. The two poles of the light induced charge carrier separation were located beyond this dashed line and the photovoltaic field was uniform (parallel field vectors) in-between these poles. In the remaining area, the photovoltaic field was non-homogenous (bend and fringe), as expected in dipolar fields. A non-trivial director pattern can be expected in the exposed test cells. From earlier works it has become clear that locally induced photovoltaic fields can lead to field induced defect creation in a layer of a nematic LC covering a Fe: $\mathrm{LiNbO}_{3}$ substrate. As initial guess, a pair of locally induced topological charges with a defect strength of $+1+-1=0$ (topologically neutral director field) was placed in an initially homeotropic director field (Fig. 2b) and the resulting director field was composed. Since the fields were locally photo induced, homeotropic alignment of the LC had to be maintained in the surrounding area of the defect pair. As indicated in the figure, placing such a pair of topological charges in a homeotropic aligned sample led to the formation of additional topological charges. In the shown schematic, the sum of the defect strengths cannot be 0 - consequently, such a director pattern cannot be formed in a real sample. Therefore, one can expect a non-trivial director pattern in the exposed samples, where additional defects, point defects or line defects should appear.
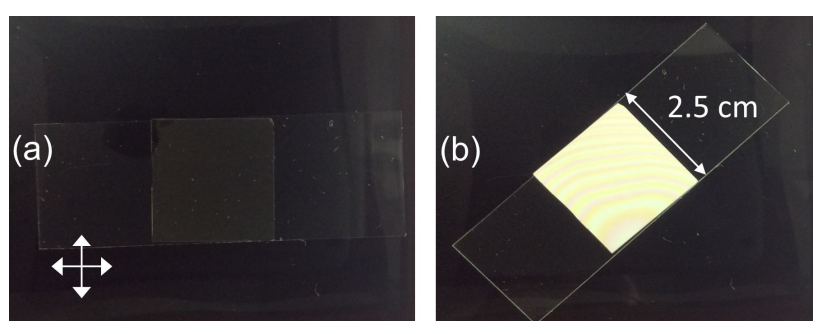

Figure 1: Photographs of $x$-cut Fe:LiNbO crossed polarizers. (a) $c$-axis parallel to one of the polarizers. (b) $c$-axis oriented $45^{\circ}$.

\section{Experimental Methods}

Fe: $\mathrm{LiNbO}_{3}$ substrates ( $x$-cut, Fe-doping concentration $0.03 \mathrm{~mol} \%$, optical quality polished surfaces, $1 \mathrm{~mm}$ thickness, obtained from Deltronic Crystal Industries) were cleaned by sonication in a water-surfactant dispersion, followed by cleansing with acetone. Photographs of the test cells (Fig. 1) were recorded by placing them on a 

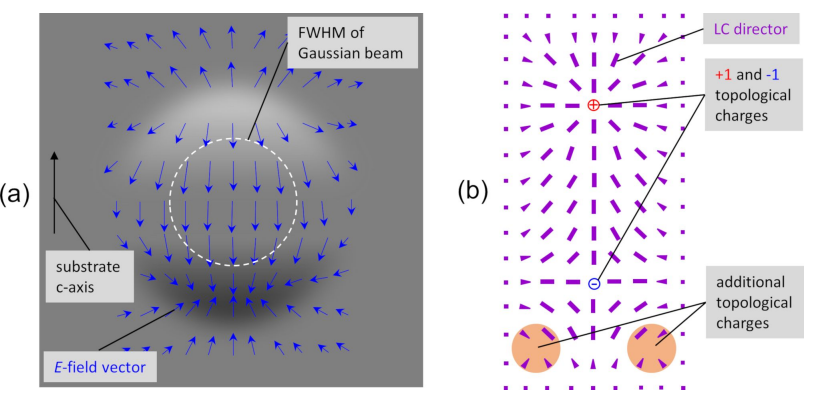

Figure 2: (a) Schematic of photovoltaic field distribution (fields in an isotropic medium) at the surface of an $x$-cut Fe:LiNbO exposed to a Gaussian laser beam. The field amplitude is shown as shaded area (gray: zero field). (b) Composed LC director pattern, where a pair of field induced topological charges was placed in an initially homeotropic director field (wedges indicate a tilted director). In the area near the negative topological charge, formation of additional topological charges(indicated) cannot be avoided, which violates the requirement of a topologically neutral director field.

cold light source equipped with crossed polarizers and using a smart phone camera. Two Mylar-film spacers were placed on the rubbed $\mathrm{Fe}: \mathrm{LiNbO}_{3}$ substrates and covered with indium tin oxide (ITO) coated cover glass, coated with lecithin as anchoring agent to provide vertical alignment of the LC. These samples were filled via capillary action by placing a droplet of the LC MLC-2087 (obtained from Merck $\mathrm{KGaA}$ ) at the edge of the covering glass plates. The LC flowed in the gap between the Fe: $\mathrm{LiNbO}_{3}$ substrates and ITO-glass. When the hybridized samples were completely filled, excess LC was removed (tissue or a disposable pipette). A cell gap $d \approx 30 \mu \mathrm{m}$ was measured with a precision digital micrometer. MLC-2087 had positive dielectric anisotropy of $\Delta \epsilon=13.31$ (Habibpourmoghadam, Jiao, et al., 2017) and a birefringence of $\Delta \mathrm{n}=0.076$ (Habibpourmoghadam, Jiao, et al., 2017).

The samples were exposed in a modified inverted optical polarized microscope equipped with CMOS digital camera (TheImagingSource, DFK MKU120-10x22) and a removable edgepass filter with a cut-on wavelength of $550 \mathrm{~nm}$ (Thorlabs FELH0550). A focused diode laser beam with a wavelength of $532 \mathrm{~nm}$ was coupled to the beam path of the microscope to expose the samples.

This setup has been used in previous studies and a detailed descriptions were published in (Habibpourmoghadam, Lucchetti, et al., 2017; Habibpourmoghadam, Jiao, et al., 2017).

The focused diode laser beam was investigated in the sample plane with a beam profiler (Spiricon SP620U) and a Gaussian intensity distribution with a full width at half maximum (FWHM) of $14.5 \mu \mathrm{m}$ was seen.

\section{Results}

The samples initially (beam shutter closed) appeared dark (Fig. 3a). Upon opening the beam shutter, LC realignments were induced and the samples locally switched to a transmissive state (Fig. 3b through d). The transition from dark to a transmissive, bright state, was spatially more expanded than the beam focus and grew continuously until a steady state was reached after $10 \mathrm{~s}$ had passed. Birefringent stripes were seen in the bright pattern and it had low symmetry (no rotational symmetry). The pattern appeared split in two areas, one left of the exposure beam and one right of the exposure beam (Fig. 3b). This split seems caused by the substrates polar axis. As indicated in (Fig. 3c), the pattern - in first approximation - could be described with a mirror axis in the sample plane, perpendicular to the substrates polar axis. However, more detailed studies (following section) revealed, this mirror axis was not present (also it can be helpful to describe the visual appearance of the pattern roughly).
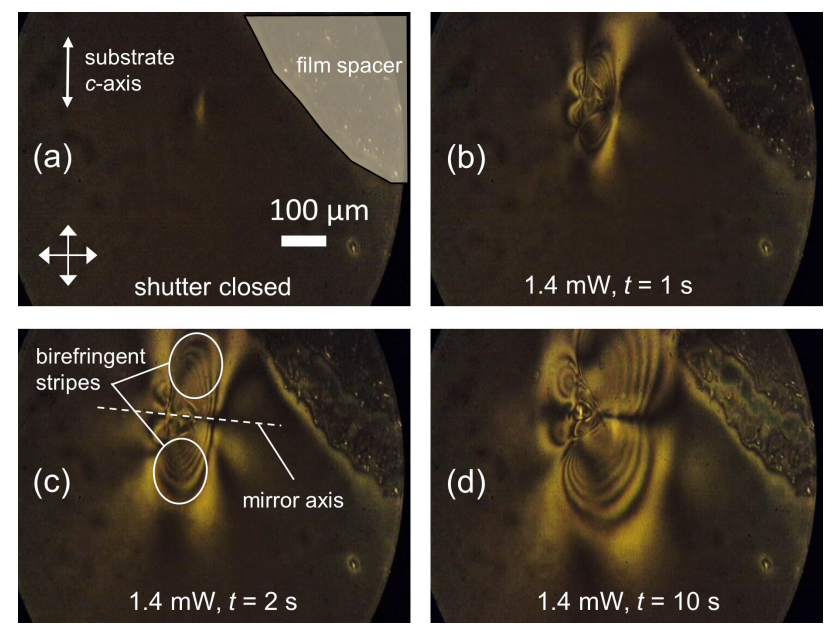

Figure 3: Light induced defect formation in vertically aligned photovoltaic LC test cell with $x$-cut substrate (polarized optical micrographs). (a) Beam shutter closed. (b through d) Recorded after the shutter was opened (recorded at various exposure times).

The observed patterns (Fig. 4) were studied in detail, in order to learn about the nature of the induced director realignment. The pattern shown in Fig. 4a can be divided in three sections. In each of these sections, birefringent stripes with different shapes were seen. The birefringent stripes in section 1 were bent. In section 2, they were parallel and in section 3, they were bent again but the pattern here was more expanded than in section 1 . In section 1 and 2, three dark stripes were seen, respectively. In section 3, 5 
dark stripes were seen. With the birefringence of MLC 2087, $\Delta n=0.076$, the layer thickness $d=30 \mu \mathrm{m}$, and a wavelength $\lambda=0.6 \mu \mathrm{m}$, one can estimate the maximum number of dark stripes: $\Delta n \cdot \frac{d}{\lambda} \approx 3$ to 4 . Well-separated stripes appear if the director tilts continuously over a width of several $\mu \mathrm{m}$. In the area far away from the exposure spot center, the director was vertically aligned. Near the exposure spot center, the director was aligned parallel to the local field direction in the plane of the test cell. This half rotation of the director was seen in area 1. Here, 3 neighboring stripes were seen, which was indicative for one half twist. In area 3, 5 stripes were observed. Area 2 and 3 were separated by a line defect (Fig. 4b): Here, the LC was vertical aligned. The director was also vertically aligned in the area far away from the exposure spot center. In area 3 , the director undergoes two half twists in radial direction, as indicated (Fig. 4b). In area 2, the director undergoes one half twist (three stripes). The director field is best described by structure of two point defects, one with positive topological charge (marked with red colored circle and + ) and one with negative topological charge (marked with blue colored circle and -). These 2 point defects were interconnected with a defect line (vertical director). The separation of the point defects was $\approx 100 \mu \mathrm{m}$. As in the composed pattern shown in Fig. 2b, most likely, these point defects were pinned at the two poles of the light induced charge carrier separation at the substrate surface and the local director had a tendency to follow the local field direction. In previous studies (Habibpourmoghadam, Jiao, et al., 2017), non-homogeneous fields led to defect formation selectively in samples, where the initial alignment of the LC and polar axis of the substrates were perpendicular. While a single umbilic defect and various line defects were created in these previous studies with $z$-cut substrates, exposure of $x$-cut substrates led to field induced formation of a pair of point defects. The director pattern in the exposed samples is indicated in Fig. 4b. In contrast to the composed director pattern (Fig. 2b), a narrow defect line occurred in the exposed sample (vertical director, separating areas 2 and 3 in Fig. 4a) and the director pattern in area 3 corresponded to a second, broad line defect. Both line defects were pinned to the 2 point defects seen. As in the composed director pattern, the director was parallel to the local electric field vector in-between these point defects. Moreover, the director also followed the direction of electric field in both areas above the positive topological charge and below the negative topological charge. In the composed director pattern, the director distribution near the negative topological charge would be perpendicular to the electric fields, locally. Therefore, the observed pattern was more suited to minimize the overall elastic energy in the exposed sample than would have been possible in the composed director pattern.
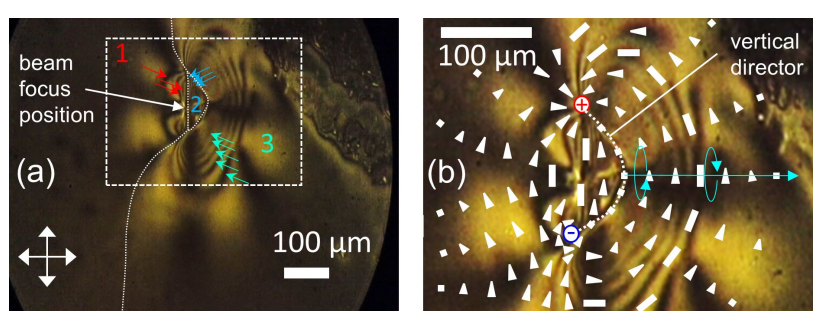

Figure 4: (a) Oberserved defect pattern. Image divided in three sections by a dashed line. Birefringent stripes (dark ones) were highlighted with arrows. (b) Magnification with added schematic of director field pattern (wedges indicate out of plane tilt of the LC director).

The evolution of the light induced pattern formation was investigated. A steady state was reached after $10 \mathrm{~s}$ exposure time. A rise time of $10 \mathrm{~s}$ is unusual for electrooptic responses in a nematic LC: The Fredericks transition can have rather slow response times - but typically in the condition of very weak addressing fields, selectively. Such weak addressing fields can only lead to slight director deformations. In the studied samples, distinct director deformations were seen. Accordingly, the observed response time of $10 \mathrm{~s}$ was a measure for the build-up time of the photo generated photovoltaic fields in the substrates rather than for the director deformations in the LC.

Images recorded during the first second are shown in Fig. 5. As seen in the figure, the shape of the pattern evolved very quickly and then grew continuously. Two birefringent stripes were seen after $0.2 \mathrm{~s}$ exposure time, 4 after $0.4 \mathrm{~s}$ in the right area of the pattern. If the laser shutter was closed, the LC relaxed back into the initial, vertically aligned director field. The response of the LC was thus fully reversible. The relaxation times $t_{\text {off }}$ increased with increasing laser power (Fig. 6) from 5 to 20 seconds.

The power of the exposure beam was varied and a threshold exposure power of $\approx 0.36 \mathrm{~mW}$ was seen. The focused laser spot had a FWHM of $14.5 \mu \mathrm{m}$, which, for this exposure power, resulted an optical power density of $\approx 140 \mathrm{~W} / \mathrm{cm}^{2}$ in the focal plane. The threshold behavior seen was most likely caused by the electro-optic response properties of the LC: In the initial state, the director was vertically aligned. The photo generated fringe fields locally (especially in the region in-between the two poles of the photo induced charge carrier separation) had large components in the sample plane (perpendicular to the initial LC director). This is a typical situation (electric field perpendicular to the LC director) where the electro-optic re- 
alignment of an LC has a threshold electric field (typically a threshold voltage of $\approx 1 \mathrm{~V}$ is observed in a LC test cell with a cell gap of $10 \mu \mathrm{m}$ ) in LC samples.

Optical nonlinearities occurring in neat, undoped, non-hybridized nematic LCs, the giant optical nonlinearity (GON) and the light induced Fredericks transition (LIFT), have been reviewed (Tabiryan, Zel'dovich, Sukhov, 1986). In experiments addressing GON, a test cell with welldefined director orientation was exposed with a focused laser beam at an angle of incidence $\alpha>0$ (oblique incidence). If so, the LC director can be locally reoriented by the light field and this effect leads to large-enough deviations from the initial director alignment such that it can be experimentally observed at optical power densities $I$ of $\approx 50 \mathrm{Wcm}^{-2}$. This threshold-free effect is usually described by an effective constant of nonlinearity $\epsilon_{2}$ and varies the permittivity of the nonlinear optical medium by a contribution $\epsilon_{2}|E|^{2}$. Considering the angle of incidence $\alpha$, the cell gap of the LC test cell $d$, the average elastic constant of the LC $K$, and the dielectric anisotropy $\epsilon_{\|}-\epsilon_{\perp}=\Delta \epsilon$ of the LC, Tabiryan et al. (Tabiryan, Zel'dovich, Sukhov, 1986) derived the following expression for $\epsilon_{2}$ :

$$
\epsilon_{2}=\frac{4 \Delta \epsilon \sin ^{2} \alpha \cos ^{2} \alpha d^{2}}{\pi^{5} K} .
$$

The deviation of the director alignment induced via GON can for example lead to self-focusing of a divergent beam in an LC layer at oblique incidence.

In contrast to GON, the LIFT effect is characterized by a threshold intensity. Light fields above threshold can lead to pronounced director reorientations. A light induced Fredericks transition can be excited at normal incidence with a laser beam of higher optical power. From the threshold electric field $E_{t h}$ of the Fredericks transition of a nematic LC:

$$
E_{t h}=\frac{\pi}{d} \sqrt{\frac{K}{\epsilon_{0} \Delta \epsilon}},
$$

(where $K$ is the average elastic constant of the LC) the condition $\frac{1}{2} c_{o} \epsilon_{0}|E|^{2}=I$ (with the speed of light $c_{0}$ and the electric permittivity of the vacuum $\epsilon_{0}$ ), and suitable geometric considerations, Tabiryan et al. (Tabiryan, Zel'dovich, Sukhov, 1986) derived the threshold intensity for LIFT transitions:

$$
I=c_{0} K\left(\frac{\pi}{d}\right)^{2} \frac{\epsilon_{\|}}{\Delta \epsilon \sqrt{\epsilon_{\perp}}} .
$$

Based on their well-explained theoretical considerations and the experiments reviewed in the reference, Tabyrian et al. reported a typical threshold optical power density of $300 \mathrm{Wcm}^{-2}$ for LIFT in a nematic LC.
Photovoltaic fields at the surfaces of field generating substrates with an in-plane $c$-axis have so far only been calculated via numerical simulations (Gazzeto et al., 2016, Arregui et al. 2014). It was shown (Gazzeto et al., 2016) that these surface near fields at the surfaces of $x$-cut Fe: $\mathrm{LiNbO}_{3}$ can easily reach field strength of $10^{5} \mathrm{~V} / \mathrm{m}$ (in these simulations, the internal electric field inside the substrates was set to $10^{7} \mathrm{~V} / \mathrm{m}$ ). Such field strength of surface near fields are of course high enough to result LC realignments and it would be desirable to investigate the coupling of the photo generated fields and the LC director, numerically.

The experiments revealed that pronounced, field induced realignments of the LC director and field induced defect creation occurred in the studied samples at normal incidence and at optical power densities $>140 \mathrm{~W} / \mathrm{cm}^{2}$ (comparable to the power density required for GON experiments) but led to even higher responses (creation of line defects) than would be possible in LIFT experiments.
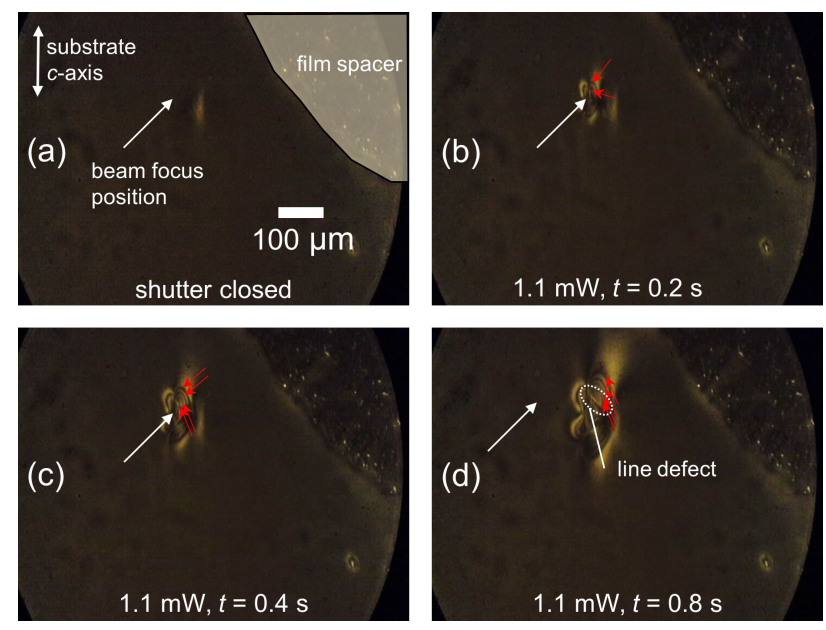

Figure 5: Evolution of the induced defect (time dependence). Birefringent stripes are highlighted with red colored arrows.

In conclusion, light induced responses were studied in hybridized LC test cells with $x$-cut photovoltaic substrates via imaging. From the recorded patterns it could be revealed, a defect structure was created upon exposure. The LC was locally fully realigned from the vertical alignment in the initial state to uniform, parallel alignment near the exposure spot center. Two coupled point defects were seen. One can speculate that the separation of these point defects corresponds to the light induced separation of the poles of the charge carrier separation in the substrates. The point defects were interconnected by a line defect (vertical director alignment in a narrow line). The LC realignment was expanded and led to transmissive states. These states were brighter than the background of the ver- 


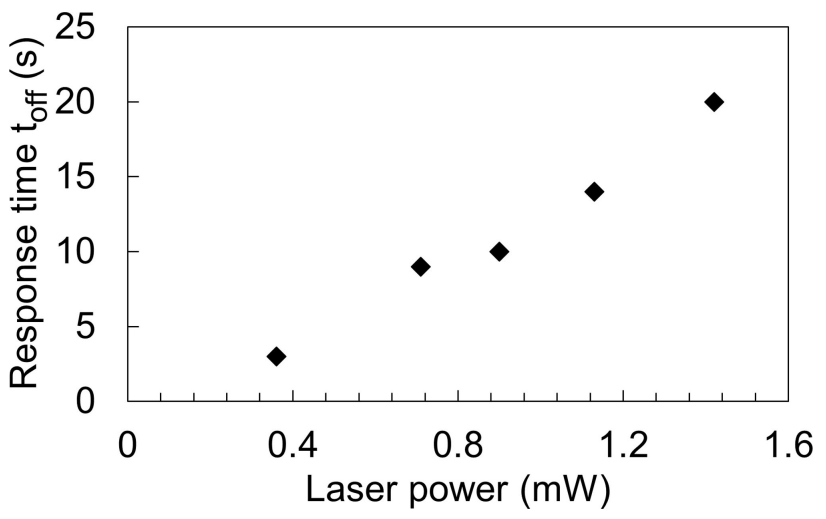

Figure 6: Relaxation time vs. laser power of the exposure beam.

tically aligned cell. The results are interesting since light induced defects with positive topological charge are useful in experiments were LCs are exploited to enhance the effectivity of optical tweezers for particle manipulation. The threshold optical power density $\left(140 \mathrm{~W} / \mathrm{cm}^{2}\right)$ seen in the hybridized samples was lower than would be required in LIFT experiments and led to much higher responses than usually observed in both LIFT and GON experiments in non-hybridized samples of a neat, nematic LC.

\section{Funding}

Funding by the US Air Force Office of Scientific Research (AFOSR) through the European Office of Aerospace Research \& Development (EOARD) grant FA9550-18-1-7002 and funding by the German Research Council (DFG) grant LO 1922/4-1 are gratefully acknowledged.

\section{References}

Arregui, C., Ramiro, J. B., Alcazar, A., Mendez, A., Munoz-Martinez, J. F., \& Carrascosa, M. (2015). Comparative theoretical analysis between parallel and perpendicular geometries for $2 \mathrm{D}$ particle patterning in photovoltaic ferroelectric substrates. Journal of the European Optical Society: Rapid Publications, 10, 15026. https://doi. org/10.2971/jeos.2015.15026

Arregui, Cándido, Ramiro, J. B., Alcázar, Á., Méndez, Á., Burgos, H., García-Cabañes, Á., \& Carrascosa, M. (2014). Optoelectronic tweezers under arbitrary illumination patterns: theoretical simulations and comparison to experiment. Optics Express, 22(23), 29099. https://doi.org/10.1364/OE.22.029099

Carns, J. L., Cook, G., Saleh, M. A., Serak, S. V., Tabiryan, N. V., Basun, S. A., \& Evans, D. R. (2006). Photovoltaic Field-Induced SelfPhase Modulation of Light in Liquid Crystal Cells. Molecular Crystals and Liquid Crystals, 453(1), 83-92. https://doi.org/10.1080/ 15421400600651757

Carns, Jennifer L., Cook, G., Saleh, M. A., Serak, S. V., Tabiryan, N. V., \& Evans, D. R. (2006). Self-activated liquid-crystal cells with pho- tovoltaic substrates. Optics Letters, 31(7), 993. https://doi.org/10. 1364/OL.31.000993

Carrascosa, M., García-Cabañes, A., Jubera, M., Ramiro, J. B., \& Agulló-López, F. (2015). LiNb03: A photovoltaic substrate for massive parallel manipulation and patterning of nano-objects. $A p$ plied Physics Reviews, 2(4), 040605. https://doi.org/10.1063/1. 4929374

Esseling, M., Zaltron, A., Argiolas, N., Nava, G., Imbrock, J., Cristiani, I., ... Denz, C. (2013). Highly reduced iron-doped lithium niobate for optoelectronic tweezers. Applied Physics B, 113(2), 191-197. https://doi.org/10.1007/s00340-013-5456-8

Fan, B., Li, F., Chen, L., Shi, L., Yan, W., Zhang, Y., ... Chen, H. (2017). Photovoltaic Manipulation of Water Microdroplets on a Hydrophobic LiNb0 3 Substrate. Physical Review Applied, 7(6), 064010. https://doi.org/10.1103/PhysRevApplied.7.064010

García-Cabañes, A, Blázquez-Castro, A., Arizmendi, L., Agulló-López, F. \& Carrascosa, M. (2018). Recent Achievements on Photovoltaic Optoelectronic Tweezers Based on Lithium Niobate. Crystals, 8(2), 65. https://doi.org/10.3390/cryst8020065

Gazzetto, M., Nava, G., Zaltron, A., Cristiani, I., Sada, C., \& Minzioni, P. (2016). Numerical and Experimental Study of Optoelectronic Trapping on Iron-Doped Lithium Niobate Substrate. Crystals, 6(10), 123. https://doi.org/10.3390/cryst6100123

Habibpourmoghadam, A., Jiao, L., Reshetnyak, V., Evans, D. R., \& Lorenz, A. (2017). Optical manipulation and defect creation in a liquid crystal on a photoresponsive surface. Physical Review E, 96(2), 022701. https://doi.org/10.1103/PhysRevE.96.022701

Habibpourmoghadam, A., Lucchetti, L., Evans, D. R., Reshetnyak, V. Y., Omairat, F., Schafforz, S. L., \& Lorenz, A. (2017). Laser-induced erasable patterns in a $\mathrm{N}^{*}$ liquid crystal on an iron doped lithium niobate surface. Optics Express, 25(21), 26148. https://doi.org/10. 1364/OE.25.026148

Lucchetti, L., Kushnir, K., Ciciulla, F., Zaltron, A., Bettella, G., Pozza, G.,... Simoni, F. (2016). All-optical phase shifter with photovoltaic liquid crystal cell. In I. C. Khoo (Ed.) (p. 99400G). https://doi.org/ $10.1117 / 12.2235580$

Lucchetti, L., Kushnir, K., Reshetnyak, V., Ciciulla, F., Zaltron, A., Sada, C., \& Simoni, F. (2017). Light-induced electric field generated by photovoltaic substrates investigated through liquid crystal reorientation. Optical Materials, 73, 64-69. https://doi.org/10. 1016/j.optmat.2017.08.004

Lucchetti, L., Kushnir, K., Zaltron, A., \& Simoni, F. (2016). Liquid crystal cells based on photovoltaic substrates. Journal of the European Optical Society: Rapid Publications, 11, 16007. https://doi.org/10. 2971/jeos.2016.16007

Tabiryan, N. V., Zel'dovich, B. Y., Sukhov, A. V. (1986). The orientational optical nonlinearity of liquid crystals. Molecular Crystals and Liquid Crystals, 136, 1-139. https://doi.org/10.1080/ 00268948608074569

Villarroel, J., Burgos, H., García-Cabañes, Á., Carrascosa, M., Blázquez-Castro, A., \& Agulló-López, F. (2011). Photovoltaic versus optical tweezers. Optics Express, 19(24), 24320. https://doi.org/ 10.1364/OE.19.024320

Volk, T. (2010). Lithium niobate: defects, photorefraction and ferroelectric switching. Springer. ISBN-13: 978-3540707653. 